\title{
Evaluation of the Involvement of the Tempromandibular Joint in Patients with Psoriasis using Computed Tomography for Detection of Psoriatic Arthritis Changes.
}

\author{
Hajer Ibrahim \\ B.D.S, Msc.oral medicine(U.S.A)
}

Muntaha fawzi salih

ABSTRACT

B.D.S.

Background: Tempromandibular disorders is a collective term, embracing several clinical roblems involving the muscles of mastication, temporomandibular joint (TMJ), or both. Tempromandibular disorders is a symptom complex rather than a single condition. TMDs form a cluster of related disorders with common symptoms which include localized pain, limited or asymmetric movement, and clicks or grating on opening.Psoriatic arthritis is a chronic inflammatory arthropathy that affects patients with psoriasis. The clinical findings for Tempromandibular PSA are pain, tenderness, limitation of movement, joint stiffness, clicking as the jaw is opened or closed, difficulty in opening the mouth, locking of the jaw, and crepitations. These findings are essentially the same as for myofascial pain/dysfunction ${ }^{(3)}$. The objective of the study were to determine the extent of tempromandibular joint involvement in patients with psoriatic arthritis and to evaluate the correlation between clinical findings and radiographic finding.

Materials and method: In this study 98 patients were selected ,50 male and 48 female, their age ranged between(18-68)years. They were collected at $\mathrm{Al}$-yarmook teaching hospital/department of.They were diagnosed as having psoriatic arthritis by a dermatologist according to (Moll and Wright diagnostic criteria 1973) ${ }^{(11)}$. These patients were subjected to rheumatoid factor test RF to exclude the presence of rheumatoid arthritic disease, then the patients were exposed to CT scanning for the Tempromandibular joint in Al-Yarmook teaching hospital for screening the involvement of TMJ with psoriatic arthritis by the presence of any radiographic changes such as erosion, flattening,osteophyte and sclerosis in the condylar head.

Results: The result of the study showed that the mean age of psoriatic arthritic patients in TMJ was (44.2) years, and female percentage was (48.4\%) and male was(51.6\%). Psoriatic arthritis in TMJ showed high significance in the unilateral side complaining $(100 \%)$ and itwas significant in the positive family history (54.8\%), and non significant in the right/left ratio (41.9\%). Oligo type was found in twenty six patients (83.9\%) with significant p-value. Clinically: all symptoms including clicking, tenderness and tempromadibular joint pain were highly significant, limitation showed (54.8\%), TMJ pain showed (64.5\%) and tenderness showed (54.8\%). Radiologically: CT scan showed sensitivity for erosion in TMJ (54.8\%), for osteophyte (32.3\%), for condylar flattening (16.1\%) and for sclerosis (9.7\%) while specificity for all were (100\%). Association between clinical and radiological changes showed non significant correlation.

Conclusions: Psoriatic arthritis of TMJ is unilateral (oligo type).Female/ male ratio is about 1:1. About $50 \%$ of patients have limitation in mouth opening. Most of the patients have positive family history. Duration of the disease is related to the development of psoriatic arthritis in TMJ. Plaque psoriasis is the most associated type with the psoriatic arthritis in TMJ. Most of patients with psoriatic arthritis show psoriatic nail changes. The most radiographic findings found in patients with psoritic arthritis was erosion in the condylar head. There is association between clinical and radiologic findings for psoriatic arthritis in TMJ.

(Keywords:Tempromandibular joint, Tempromandibular disorders, psoriatic arthritis, Computed tomography).

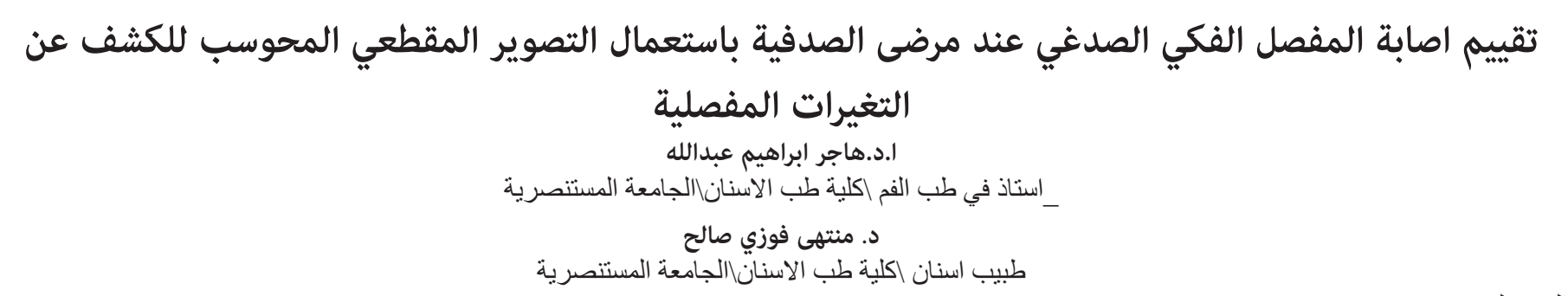

اضطر ابات المفصل الفكي الصدغي تشير الى مصطلح مركب يحتوي على عدة حالات نتضمن المفصل الصدغي,التركيبات المحيطة او كلاهما.

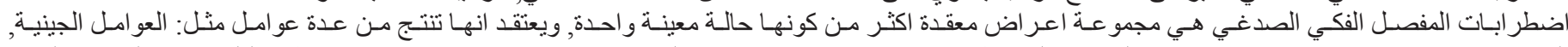

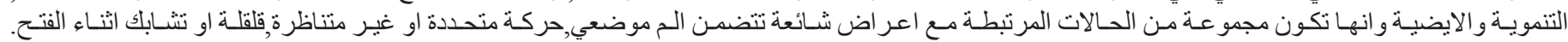

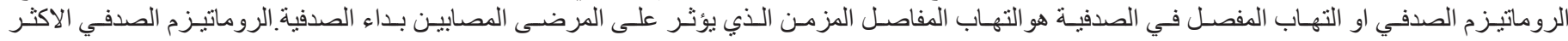

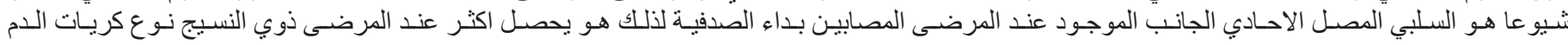

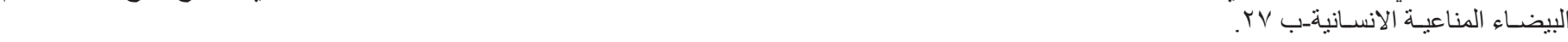

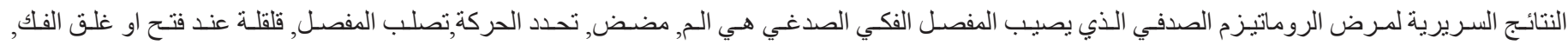

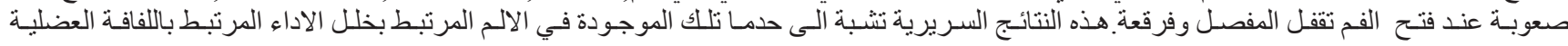
التحديد مدى شمول البحث المفصل الفكي الصدغي لاى المرضى المصابين بالتهاب المفصل في الصدفية. 


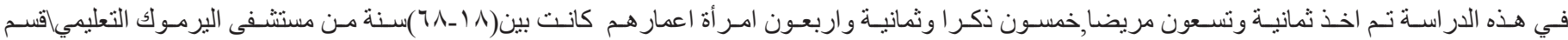

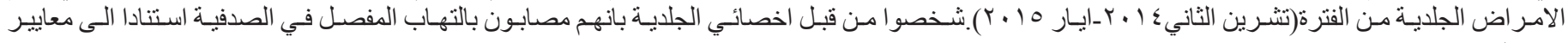

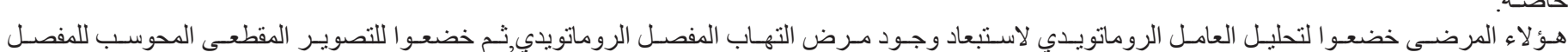

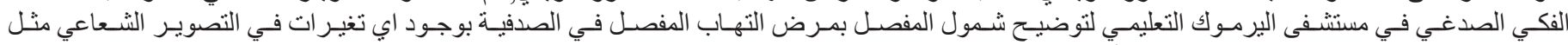

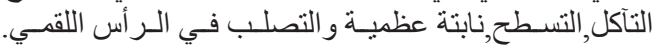

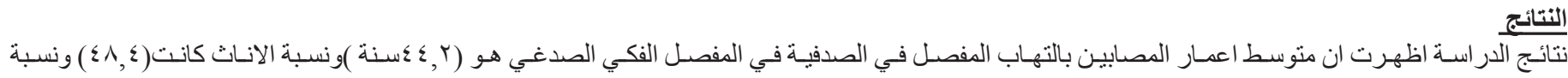

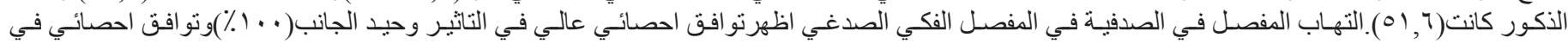

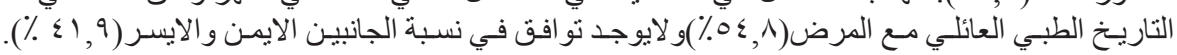

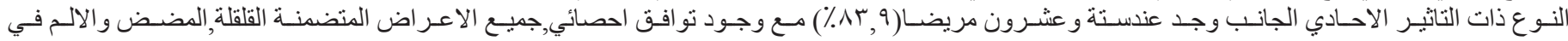

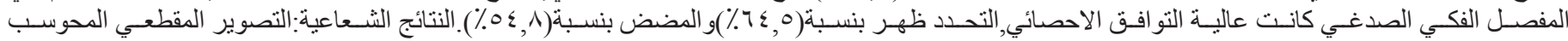

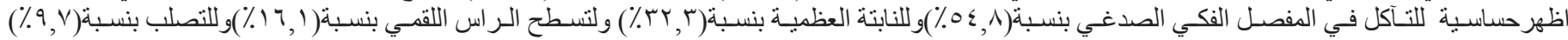

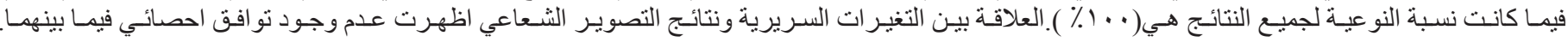

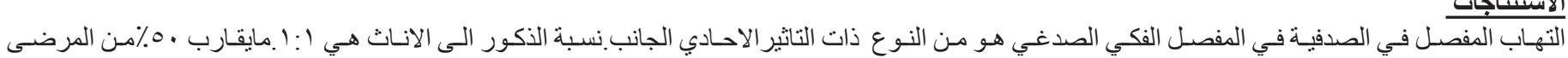

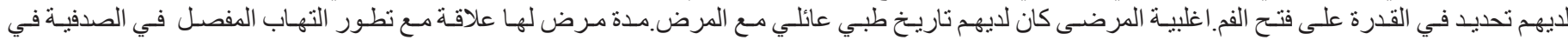

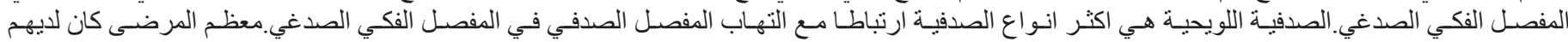

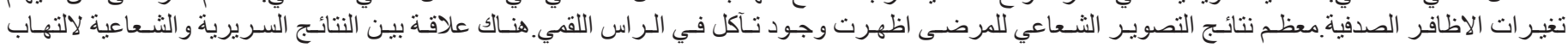

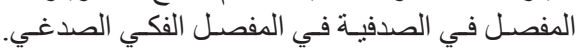

\section{INTRODUCTION}

Psoriasis is a chronic, autoimmune disease that appears on the skin. It is not contagious,yet is the most common autoimmune disease in the United states. Psoriasis has been linked to other serious health conditions, such as cardiovascular disease and depression. About 30 percent of people with psoriasis develop psoriatic arthritis, which causes pain, stiffness and swelling in and around the joints ${ }^{(1)}$.

Psoriatic arthritis can develop at any age. Genes, the immune system, and environmental factors are all believed to play a role in the onset of the disease. Arthritis is not correlated with the extent of skin disease $^{(2)}$. psoriatic arthritis result in destructive arthritis in which the inflammatory process leads to bony erosion and loss of joint architecture

Psoriatic arthritis (PsA) has historically been considered a milder rheumatic disease not yielding significant clinical damage. However, recent studies have shown that PsA can be deforming and debilitating and that joint damage can be severe ${ }^{(5)}$. Traditionally, joint damage has been recorded using plain radiographs. Characteristic radiographic features of PsA include joint erosions, joint space narrowing, bony proliferation including periarticular and shaft periostitis, osteolysis including "pencil in cup" deformity and acro-osteolysis, ankylosis, spur formation, and spondylitis (4).

In $80 \%$ of patients, psoriasis usually precedes the development of arthritis Treatment is by using anti-inflammatory agents, anti-malarials, and adding methotrexate ${ }^{(15)}$.Treating the skin alone seems to have little impactonjoint disease, and the relationship between skin and joints is still unclear. Psoriatic arthritis of the temporomandibular joint(TMJ) was described in 1965, and in the twenty cases reported the TMJ affection has been part of polyarthritis in patients who have suffered from cutaneous psoriasis for years. Clinically, the temporomandibular joint has presented symptoms of chronic arthritis (pain, TMJ tenderness and restricted movement). Radiologic imaging has revealed abnormalities in condyle position, erosion, Osteoporosis ${ }^{(6)}$.

\section{SUBJECT,MATERIALS AND METHOD}

Patients were diagnosed as having psoriasis by the dermatologist, then Moll and Wright diagnostic criteria were applied to these patients for diagnosis of psoriatic arthritis. If tow of the first four criteria and the fifth one were achieved the patient diagnosed as having PsA. an inflammatory arthritis (peripheral arthritis and/or sacroiliitis or spondylitis).

- The presence of psoriasis.

- Nail involvement such as pitting and separation from the nail bed (onycholysis), as well as yellowpink discoloration (the oil-drop sign).

- Sausage digits (dactylitis).

- The (usual) absence of serological tests for rheumatoid factor (11).

Clinical examination: The clinical examination started with examination of psoriatic skin lesions, then examination of nails for psoriatic nail changes.

\section{TMJ pain on palpation:}

The tip of the index finger was placed over the 
lateral aspects of both joint area and slight pressure was applied, pain or tenderness was recorded in a static position or during opening and closing of the mouth. The same procedure was repeated to examine the posterior aspect of the TMJ via external audiatory meatus using the index and middle fingers, pain on movement or tenderness was recorded whenever palpation resulted in reflex or the patient reported a subject discomfort ${ }^{(13)}$.Computed tomography scans were carried on Philips Brilliance CT. Bilateral tempromandibular joint CTscans were obtained for all 98 patients.All CT scans were evaluated in details by Radiologist .Each condyle was evaluated for for changes like erosion,flattening, osteophyte formation and sclerosis. The clinical data then was correlated with the CT scan findings for each joint and subjected to statistical evaluation.

\section{RESULT}

The result of the study showed that the mean age of psoriatic arthritic patients in TMJ was (44.2) years, and female percentage $(48.4 \%)$ and male $(51.6 \%)$. Psoriatic arthritis in TMJ showed highly significant in the unilateral side complaining $(100 \%)$ and its significant in the positive family history (54.8\%) and non significant the right/left ratio (41.9\%). Oligo type was founded in twenty six patients $(83.9 \%)$ with significant p-value, clinically: all symptoms including clicking, tenderness and tempromadibular joint pain were highly significant, limitation showed (54.8\%), TMJ pain showed (64.5\%) and tenderness showed (54.8\%).radiologically: CT scan showed sensitivity for erosion in TMJ $(54.8 \%)$, for osteophyte $(32.3 \%)$, for condylar flattening (16.1\%) and for sclerosis $(9.7 \%)$ while specificity for all were (100\%). Association between clinical and radiological changes showed non significant correlation

Table (1) :Description of patient's age and statistical analysis

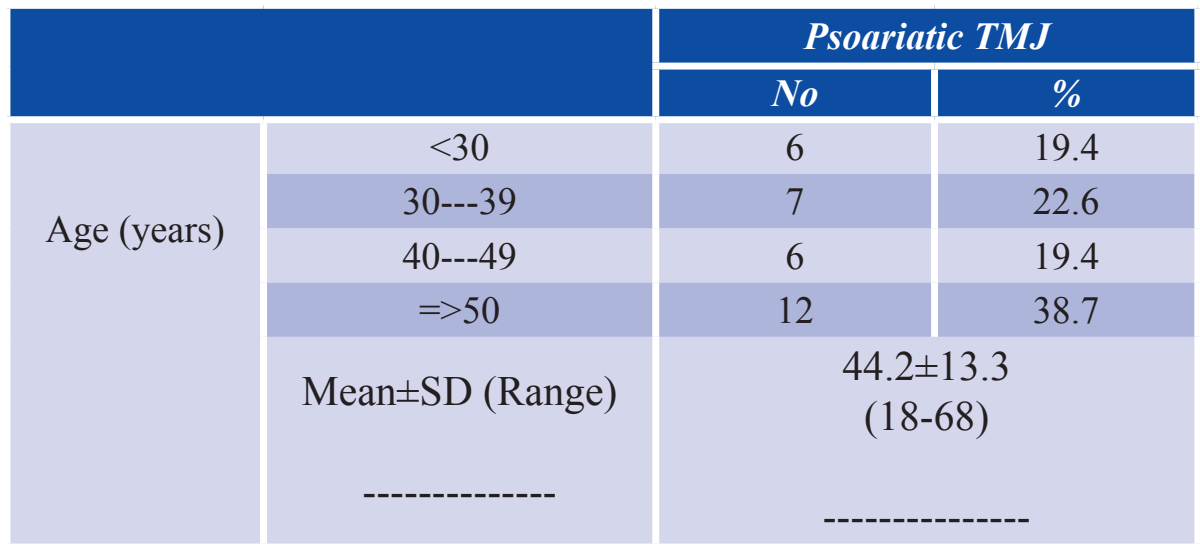

\begin{tabular}{|c|c|c|}
\hline \multicolumn{2}{|c|}{ Psoariatic arthritis } & P value \\
\hline No & $\%$ & \% \\
\hline 7 & 10.4 & 0.645 \\
\hline 17 & 25.4 & \\
\hline 17 & 25.4 & \\
\hline 26 & 38.8 & \\
\multicolumn{2}{|c|}{$44.5 \pm 11.4$} \\
(19-61)
\end{tabular}

Figure (1) :The clinical presentation

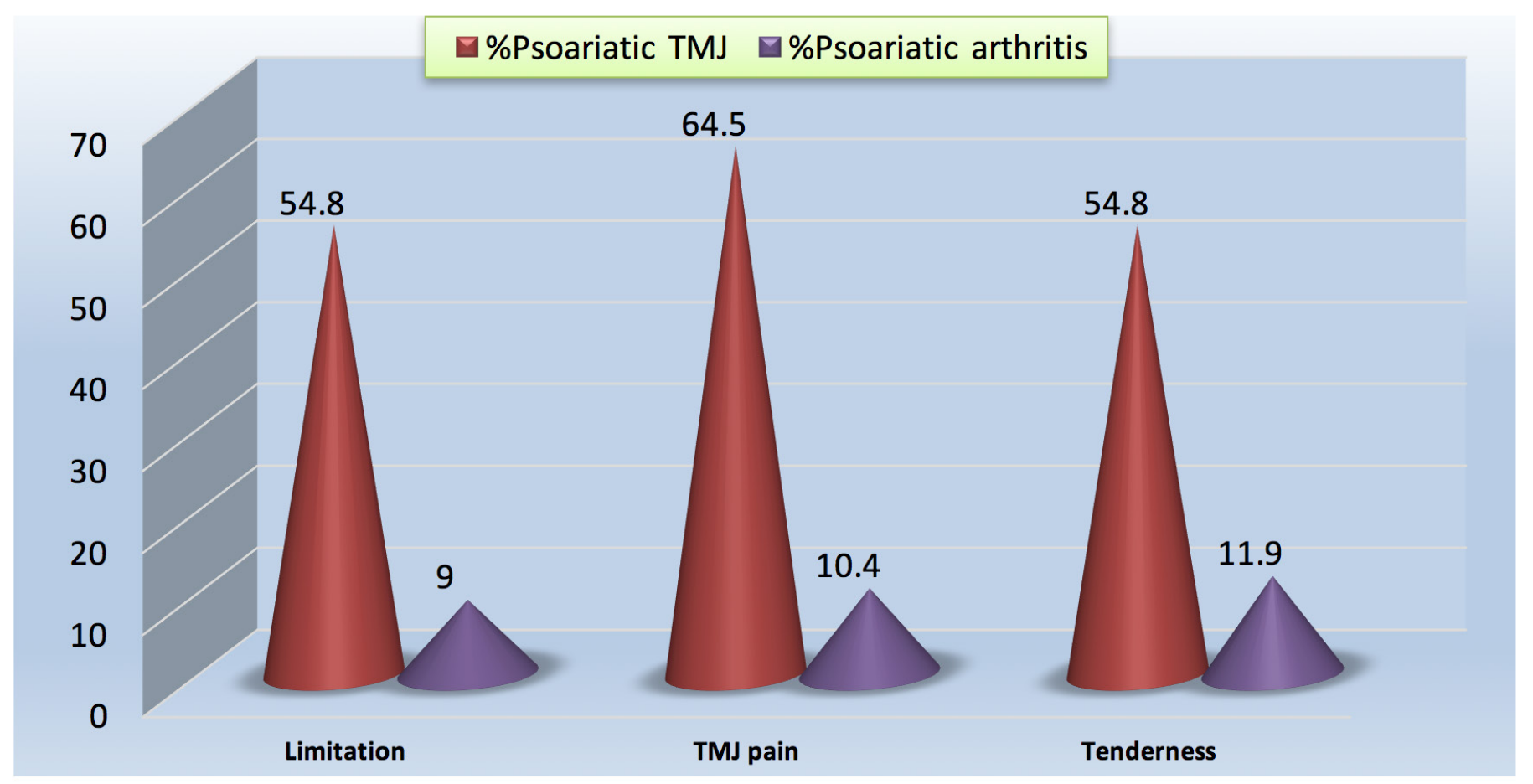


Figure (2) :The percentage of type and associatedfeatures.

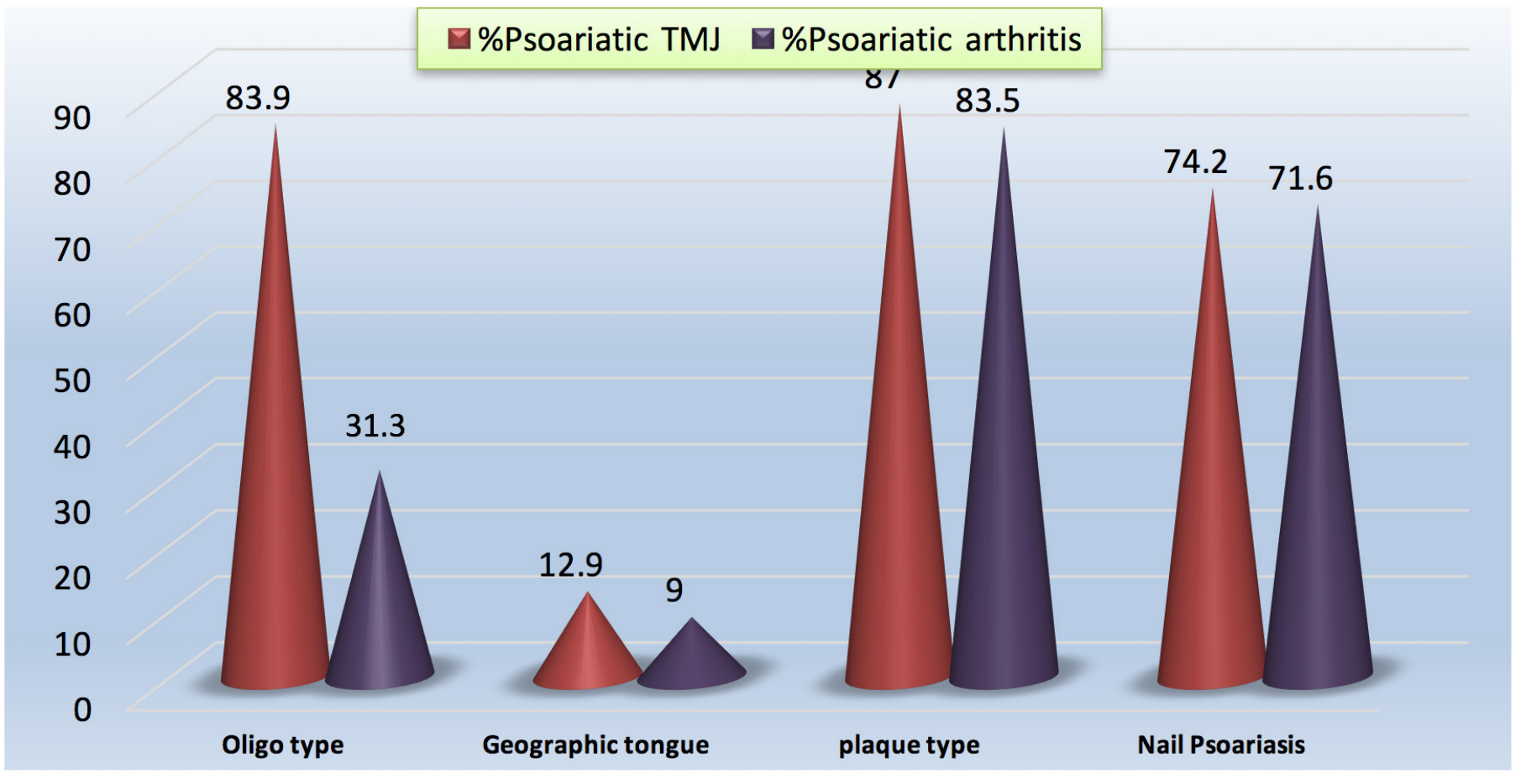

Table (2) :The radiological findings of $\mathrm{CT}$.

\begin{tabular}{|c|c|c|c}
\hline \multirow{2}{*}{ CT findings } & & Psoariatic TMJ & $\begin{array}{c}\text { Psoariatic ar- } \\
\text { thritis }\end{array}$ \\
\cline { 3 - 4 } & & No & No \\
\hline \multirow{2}{*}{ Erosion } & Yes & 17 & - \\
& No & 14 & 67 \\
\hline \multirow{2}{*}{ Osteophyte } & Yes & 10 & - \\
\hline \multirow{2}{*}{ Flattening } & No & 21 & 67 \\
\hline \multirow{2}{*}{ Sclerosis } & Yes & 5 & - \\
& No & 26 & 67 \\
\hline
\end{tabular}

*Significant difference in proportions using Pearson Chi-square test at 0.05 level

Association between type of psoriatic arthritis and radiological changes.

\begin{tabular}{|c|c|c|c|c|c|c|}
\hline \multirow{2}{*}{\multicolumn{2}{|c|}{ CT findings }} & \multicolumn{2}{|c|}{ Oligo } & \multicolumn{2}{|c|}{ Polv } & \multirow{2}{*}{ Pvalue } \\
\hline & & No & $\%$ & No & $\%$ & \\
\hline \multirow{2}{*}{ Erosion } & Yes & 14 & 53.8 & 3 & 60.0 & 0.800 \\
\hline & No & 12 & 46.2 & 2 & 40.0 & \\
\hline \multirow{2}{*}{ Osteophyte } & Yes & 7 & 26.9 & 3 & 60.0 & 0.147 \\
\hline & No & 19 & 73.1 & 2 & 40.0 & \\
\hline \multirow{2}{*}{ Flattening } & Yes & 5 & 19.2 & - & - & - \\
\hline & No & 21 & 80.8 & 5 & 100.0 & \\
\hline \multirow{2}{*}{ Sclerosis } & Yes & 3 & 11.5 & - & - & - \\
\hline & No & 23 & 88.5 & 5 & 100.0 & \\
\hline
\end{tabular}

* Significant difference in proportions using Pearson Chi-square test at 0.05 level

\section{DISCUSSION}

In our study we found that the mean age of psoriatic arthritis in TMJ was (44.2), and for control group (44.5) which is very close to the group of 
Wilson $^{(8)}$. with mean age(43.3), while Kononen ${ }^{(7)}$ found that the mean age was (51) for both arthritic group and control group , and Green ${ }^{(9)}$ found the mean age was (46.08). Sex distribution in our study showed that male $(50.7 \%)$ and female $(49.3 \%)$ approximately the same to other studies like Wilson in $50 \%$ for each one. Other study by Kononen showed that male $(57.2 \%)$ and female $(42.8 \%)$. While other study by Green showed sex distribution (1.2:1) very close to our study.In the view of 43 cases of psoriatic arthritis involving TMJ, there is a male predilection (24:19) and the average age of onset is 43.3. TMJ lesions could occur before, during, or after other joints involvement. Zhi ${ }^{\left({ }^{10}\right)}$ found the most common clinical findings are pain in the TMJ region (27/43). TMJ tenderness to palpation (22/43), and limited mouth opening was (21/43). The unilateral involvement of TMJ in patients with psoriatic arthritis is more common than bilateral involvement (5:3), these features in agreement with the findings of Rasmussen and Bakke ${ }^{(14)}$.Moreover, some clinical studies reported that muscle tenderness on palpation and joint sounds were the most frequent symptoms in TMJ, while restricted mouth opening was not prominent in psoriatic arthritis patients. It could be inferred that the symptoms of psoriatic arthritis affecting the TMJ are nonspecific. While in our study All patients examined clinically and it's highly significant in psoriatic arthritis in TMJ for limitation, TMJ pain and tenderness. Limitation showed (54.8\%), TMJ pain showed $(64.5 \%)$ and tenderness showed $(54.8 \%)$,very close to the result obtained by Zhi. In our study Psoriatic arthritis in TMJ showed highly significant in the unilateral side complaining (100\%) and its significant in the positive family history $(54.8 \%)$ and non significant in the right/left ratio (41.9\%). The difference in the studies between our study and (Zhi was only the percentages in side complaining, this may be due to the variance in the sample size and the race of affected population. Wilson et al, 2009 found plaque type with $(79 \%)$ followed by guttate type, similar to our study in which we found plaque type ( $87 \%$ ) followed by guttate type.

Among previous studies $\mathbf{Z h i}$ found erosion is the most common radiographic changes in psoriatic arthritis in patients with TMJ involvement (37/63) about $(58 \%)$, which is in accordance with radiologic findings of Lundberg ${ }^{(12)}$ Other radiographic findings such as bony proliferation (22/63) about (44\%), condylar flattening ( $8 / 63)$ about $(12 \%)$ and sclerosis $(6 / 63)$ about $(9.5 \%)$, are also typical changes in TMJs of psoriatic arthritis patients. This result seems close to the result obtained in our study that $\mathrm{CT}$ scan showed sensitivity for erosion in TMJ (54.8\%), for osteophyte $(32.3 \%)$, for condylar flattening (16.1\%) and for sclerosis $(9.7 \%)$ while specificity for all were $(100 \%)$.The radiographic sign score did not correlate significantly with the duration, extent, or severity of PA Kononen. The Radiographic score correlated with the subjective symptoms score $(p<0.01)$, and one of the variables of, osteophytes, correlated with pain in the TMJ experienced at the onset of subjective symptoms of craniomandibular disorders $(p<0.01)$. The radiographic score, and especially erosions in the condyle, correlated with the clinical dysfunction score.

\section{CONCLUSIONS}

- Psoriatic arthritis of TMJ is unilateral (oligo type).

- Female/ male ratio is about 1:1.

- About $50 \%$ of patients have limitation in mouth opening.

- Most of the patients have positive family history.

- Duration of the disease is related to the development of psoriatic arthritis in TMJ.

- Plaque psoriasis is the most associated type with the psoriatic arthritis in TMJ.

- Most of patients with psoriatic arthritis show psoriatic nail changes.

- The most radiographic findings found in patients with psoritic arthritis was erosion in the condylar head.

- There is association between clinical and radiologic findings for psoriatic arthritis in TMJ.

\section{REFERENCES}

1. Taylor W, Gladman D, Helliwell P, Marchesoni A, Mease P, Mielants H. Classification criteria for psoriatic arthritis: development of new criteria from a large international study. Arthritis Rheum Aug 2006;54(8):2665-73.

2. Chandran V, Raychaudhuri SP. Geoepidemiology and environmental factors of psoriasis and psoriatic arthritis. J Autoimmun May 2010; 34(3):J314-21.

3. American Academy of Orthopaedic Surgeons, 2012.

4. Klippel JH. Primer on the rheumatic diseases. Springer Verlag. (2008)

5. Todd,Adam,and Suja Elizabeth George(Current and potential new therapies for the treatment of psoriasis) pharmaceutical Journal (vol 284) 2010.

6. American Association for Dental Research. Retrieved 6 June $r \cdot 1 r$,Temporomandibular Disorders (TMD) used in (WHO)

7. Könönen M. Craniomandibular disorders in psoriasis. Community Dent Oral Epidemiol. 1987;15:10812 .

8. Wilson FC, Icen M, Crowson CS, McEvoy MT, Gabriel SE,Maradit Kremers H. Time trends in epidemiology and characteristicsof psoriatic arthritis over 3 decades: a populationbasedstudy. J Rheumatol. In press.2009.

9. Green L, Meyers OL, Gordon W, Briggs B. Arthritis in psoriasis. Ann Rheum Dis 1981;40:366-9.

10. Zhi Hu Wang, Yan Ping Zhao, Yu Chen Ma: volume 14 
number 1, 2014.

11. Moll JMH, Wright V. Psoriatic arthritis. Semin Arthritis Rheum 1973;3:55-78

12. Lundberg M,Ericson S. changes in Tempromandibular joint in psoriasis arthropathica.acta derm venereal(stockh);1967;47,354 8. ISBN:0387356649.

13. Dalkiz, :Evaluation of temporomandibular joint dysfunction by magnetic resonance imaging,2001:80-86.
14. Rasmussen, O.C.: Semiopaque Arthrography of the Temporomandibular Joint, Stand. J. Dent. Res. 88: 521$534,1982$.

15. Paul Emery, MD, and Zoe Ash, MD, American College of Rheumatology September 2012. 